\title{
LA VISITA DE HERBERT HOOVER A CHILE Y ARGENTINA EN 1928
}

\section{HERBERT HOOVER'S VISIT TO CHILE AND ARGENTINA IN 1929}

\author{
MAURICIO JARA* \\ miara@upla.cl
}

\section{RESUMEN}

Se analiza con informaciones de prensa chilena y argentina la visita que el Presidente Electo de Estados Unidos Herbert Hoover hizo a estos dos paises antes de asumir of icialmente el cargo en Washington. Se exponen las razones que habria tenido en cuenta para realizar esta gira, los resultados que de ésta se esperaban y algunos aspectos de su estadia en Chile y Angentina en diciembre de 1928.

Palabras Claves: Hoover en Chile y Argentina, gira Sudamericana de Hoover, Ibảñez, Irigoyen, 1928.

ABSTRACT

The visit of the President Elect of the United States Herbert Hoover to Argentina and Chile before assuming office is analyzed through the study of press sources from both of the respective countries. The reasons he had for making this trip, the results that were expected from it and certain aspects of his stay in Chile and Argentina in December of 1928.

Key Words: Hoover in Chile and Argentina, Southamerican tour of Hoover, Ibáñez, Irigoyen, 1928.

La visita que efectuará el Presidente Electo de Estados Unidos Herbert Clark Hoover a algunos países latinoamericanos y a Chile y Argentina en particular, en diciembre de 1928, es uno de los acontecimientos interamericanos más importantes de la primera mitad del siglo XX. Su importancia radica no solamente en el hecho de haber sido la primera visita de un Presidente de Estados Unidos a estos dos paises sudamericanos, sino también porque a partir de esta gira se habria iniciado una nueva orientación de la política internacional estadounidense hacia Latinoamérica. Politica que en la década siguiente el Presidente Roosevelt la habría proclamado como propia y que llamó del "Buen Vecino".

Si nos remitimos al momento o circunstancia en que Hoover habria insinuado por primera vez la idea de buscar un nuevo posicionamiento para Estados Unidos en el mundo, todo parece indicar que aquello se produjo en el último discurso radial de su campaña presidencial del 5 de noviembre de 1928 cuando "dijo que esta elección sería el acontecimiento más importante que se registra desde hace muchos años, porque hemos entrado a una nueva era de acción económica y moral, no sólo en nuestro país, sino que, a lo largo de todo el mundo" 1 .

En estas breves pero significativas palabras de cierre de campaña, Hoover advertia la ungencia de encabezar cambios en la política interior y exterior de Estados Unidos y que ello, de ser necesario en el ámbito continental, requeriria tener que realizar un viaje, visita o gira hacia el centro y sur de América.

En este sentido el 'The Daily Chronicle', al comentar la elección de Hoover y sus futuras actividades, sostenía que él "ha demostrado siempre instintos de exploración, organización y creación, por lo cual no será un hombre que vaya a estar ocioso en la Casa Blanca"2.

\footnotetext{
- Magister en Historia, Centro Estudios Hemisféricos y Polares, Universidad de Playa Ancha, Chile, FONDECYT N" 1070017

1 "El Ôltimo Discurso que Pronunció Mr. Hoover", El Mercurio de Santrigo (en adelante EMS), 7 noviembre 1928, p. 12.

2 "Lo que Dicen los Diarios Británicos", EMS, 9 noviembre 1928, p. 10.
} 
El periódico español 'El Debate', se equivocaba cuando afirmaba que "el triunfo de Mr. Hoover supone la continuación de la politica imperialista en las Filipinas y en parte de la América española" ${ }^{\text {, }}$, pues el propio Presidente Electo a los dos dias de haber sido elegido se encargó de despejar esa errónea suposición y anunció que próximamente realizaría un viaje hacia Latinoamérica ${ }^{4}$.

Las reacciones del sorprendente anuncio no se hicieron esperar y rápidamente circuló en los diferentes medios de prensa latinoamericanos e internacionales que era altamente probable que el viaje de Hoover "cambie la política internacional, lo que seria altamente interesante para la América Latina" 5 .

El embajador chileno en Washington, Carlos G. Dávila, al conocer la noticia del próximo viaje del Presidente Electo Hoover, decía a los funcionarios del Departamento de Estado que el futuro "presidente hará una gira de buena voluntad" 6 para con ello demostrar que su gobierno estaba dispuesto a producir un vuelco en las relaciones hemisféricas.

En tanto el embajador argentino en Estados Unidos, Manuel E. Malbrán, sostenia que dicho viaje "será sin duda un factor eficaz para la intensificación de las buenas y amistosas relaciones actualmente existentes entre ambos paises. También dará la oportunidad al Presidente de los Estados Unidos de conocer las condiciones de nuestro pais. A la vez dará a nuestro pueblo y Gobierno la ocasión de exteriorizar sus sentimientos amistosos hacia esta gran nación"7.

Fuera de la favorable opinión que ambos diplomáticos tenian sobre la visita del futuro mandatario, lo más interesante era que podria ayudar a resolver situaciones puntuales que afectaban a los aranceles del maiz y la semilla del lino en Argentina y sobre las cebollas en Chile e incluso, levantar el embargo sanitario impuesto a las naranjas brasileñas en Estados Unidos 8 .

En general para la prensa estadounidense el "proyecto de gira de Mr. Hoover era un acto constructivo de hábil estadista, calculado con el propósito de fomentar amistades mas estrechas y aplanar las dificultades en las relaciones interamericanas" 9 .

En el diario 'La Prensa' de Nicaragua, país que conocía y estaba todavia 'soportando' la presencia militar estadounidense desde hace mucho tiempo, la próxima visita de Hoover era interpretada como "el paso mas grande dado hasta ahora en las relaciones entre los Estados Unidos y la América Latina, un viaje que marcaria una nueva era de prosperidad y paz de Nicaragua"10.

En Lima, el Canciller Rada y Gamio, dijo a la agencia de noticias United Press que la visita de Hoover permitirá que "él se dé cuenta personalmente del cuan hondo y sincero es el afecto que siente el Perú hacia su gloriosa patria y contribuirá a estrechar relaciones que siempre han unido al Perú con los Estados Unidos"11. Como también que su visita al suelo peruano constituirá "el acto panamericano de más grande trascendencia" 12 para la región sudamericana.

El diario 'News of the World' de Londres, al comentar la elección de Herbert Hoover a la Casa Blanca, afirmaba que era "muy significativo el primer acto después de su elección de visitar los países de centro y Sud-América, con el objetivo de consolidar las relaciones de Estados Unidos y los países latinoamericanos; sobre todo porque atraería a todos los paises del continente americano, con excepción de Canadá, hacia una especie de Federación"13.

Para la prensa parisina en cambio, el proyectado viaje del nuevo presidente americano "será muy favorable para que los Estados Unidos recobren su perdido prestigio"114.

\footnotetext{
"Hablan los Diarios Españoles", EMS, 9 noviembre 1923, p. 10.

"Mr. Hoover Visitari La América del Sur", EMS, 9 noviembre 1928, p. 10.

"Llegari a Intervenir Mr. Hoover en el Problema de Tacna y Arica", EMS, 11 noviembre 1928, p. 34.

"Las Paises Latinoamericanos 'Tributarán un Caluroso Recibimiento a Mr. Hocwer", EMS, 12 de noviembre de 1928, p. 12.

"El Presidente Electo de los E. Unidos. Quiere Familiarizarse con los Problemas Comunes a la Unión y la America Latina y Evidenciar su Deseo de Mutua Cooperación" (Habla el Embajador de Argentina), La Nación (en adelante LN) Buenos Aires, 10 de noviembre de I928, p. 1.

* Se Embarcará en el Acorazado Maryland Antes de Quince Dias, Probablemente, y Después de Visitar el Perú y Chile Cruzará Los Andes en un Tren Especial" (Itinerario del Viaje de Regreso), LN, Buenos Aires, 10 noviembre 1928, p. 1.

v "Los Paises Latinoamericanos Tributarin un Caluroso Recibimiento a Mr. Hoover", EMS, 12 de noviembre de 1928, p. 12.

10 "Los Paises Latinosmericanos Tributarán un Caluroso Recibimiento a Mr. Hoover" (Las Deseos de Nicaragua), EMS, 12 de noviembre de 1928, p. 12.

14 "Los Patses Latinoamericanos Tributaran un Caluroso Recibimiento a Mr. Hoover" (Habla el Canciller Peruano), EMS, 12 de noviembre de 1928, p. 12 .

12. Ibid.

13 "Los Paises Latinoamericanos Tributaran un Calaroso Recibimiento a Mr. Hoover" (Apreciaciones de un Diario Británico), EMS, 12 de noviembre de 1928, p. 12.

14 "Comentarios de la Prensa Francesa"; EMS, 13 noviembre 1928, p. 12.
}

Revista de Historia, año 18, vols. 18-19, 2008-2009, pp. 93-98. 
El 'O Jornal do Commercio' de Río de Janeiro, se quejaba de que la "victoria de los republicanos en los Estados Unidos crea un problema desde el punto de vista brasileño a causa de las conocidas teorias de Mr. Hoover con respecto a nuestra politica del café" 15 . El hecho que los productores cafeteros del Brasil tengan que lidiar con altos aranceles estadounidenses dejará al "nuevo presidente en una contradicción de su política externa con su política interna"16, y por lo mismo, aconsejaba el 'O Jornal do Commercio' que era preferible esperar antes de anticipar juicios aventurados sobre el porvenir de la nueva politica de Estados Unidos con la región latinoamericana.

Con posterioridad a la victoria del 6 de noviembre, las noticias del viaje de Hoover se sucedían con rapidez y entusiasmo inusitado. El 13 de noviembre se señalaba que el Presidente Electo acompañado de su esposa, su hijo y del experimentado diplomático en asuntos latinoamericanos y ex Embajador en Chile, Henry P. Fletcher, iniciaria su gira de veinte y seis dias el 19 de ese mes desde Palo Alto ${ }^{17}$. El que la visita tuviera como objetivo fundamental desarrollar la buena voluntad se debía a "que en varios paises sudamericanos el prestigio y popularidad de Estados Unidos están en franca decadencia. Con la politica que hemos seguido en la América Central y en las Indias occidentales - se decía en medios cercanos a Hoover -, no nos hemos hecho querer de la América Latina en general" 18 .

Al acercase la fecha del zarpe desde Estados Unidos las impresiones y sensibilidades en los países que Hoover visitaria se hicieron más intensas. A los aplausos que le prodigó una frenética multitud al despedirlo desde el puerto de San Pedro también se sumaron manifestaciones desaprobatorias de "agitadores comunistas que condenaban la política norteamericana en Nicaragua"19.

Luego de cortas visitas de estilo en Centro América y en Guayaquil ${ }^{20}$, Hoover continuó su viaje hacia el sur en el acorazado 'Maryland'. Para Hoover y sus asesores, según podemos interpretar de las informaciones periodisticas, la idea era ir desarrollando un programa de contactos más que de visitas prolongadas a fin de ganar en expectación internacional y evitar caer en expresiones o promesas que en el futuro pudieran generar conflictos o desinteligencias en la política que se pensaba llevar adelante en el hemisferio occidental.

En el Perú las impresiones iniciales y finales de la visita de Hoover contrastaron abiertamente; en Lima se pensaba que el Presidente Electo estadounidense estaria varios días como para intercambiar opiniones, trabajar en materias bilaterales, recorrer parte del pais y recibir los agasajos y festejos que se merecia ${ }^{21}$, no obstante, la breve estadía que apenas alcanzó para un banquete en su honor y prontamente se embarcó en dirección al sur, causaron gran desilusión y hasta cierto modo molestia ${ }^{22}$.

Mayor desagrado causó en La Paz el hecho que Bolivia no seria visitada y que Hoover estaba dispuesto únicamente a recibir y tener una entrevista a bordo del Maryland, en la bahía de Antofagasta, a una delegación oficial del país altiplánico ${ }^{23}$. En la mañana del 8 de diciembre Hoover recibió al Canciller boliviano Alberto Palacios y luego de intercambiar expresiones protocolares ambos concordaron en que los temas regionales eran de gran optimismo para el futuro de la confraternidad americana ${ }^{24}$.

La llegada de Hoover a Valparaiso -el San Francisco del Sur- fue de gran emotividad ciudadana y en muchos sentidos hicieron recordar a las fiestas del pasado Centenario y la todavía reciente visita del Príncipe de Gales ${ }^{25}$. En la mañana del dia 11 de diciembre Hoover se trasladó en un tren especial a Santiago, llegando a la Estación Alameda antes del mediodia, en donde era esperado por el Presidente Carlos Ibáñez y los

is "Los Paises Latinoamericanos Tributarän un Caluroso Recibimiento a Mr. Hoover" (Articulos de la Prensa Brasileria), EMS, 12 de noviembre de 1928, p. 12.

16- Ibid.

17 "El Dia de la Partida de Mr. Hoover", EMS, 13 noviembre 1928, pág. 12 y "Hoover Iniciara Hoy su Jira por los Paises de la America Latina", LN, Buenos Aires, 19 noviembre 1928. p. L.

is EMS, Idem

19 "Mr. Hoover ha iniciado su Viaje de Buena Voluntad. Manifestaciones Comunistas", EMS, 20 noviembre 1928, p. 10.

30. "El Presidente Electo de la Unión Llegara Mafiana al Golfo de Fonseca", LN, Buenos Aires, 24 noviembre 1928, pp. 1-2 y "El Presidente Electo de los Estados Unidos salio Ayer de Guayaquil para el Perú", LN, Buenos Aires, 3 diciembre 1928, p. 2.

21 "Programa de Festejos en Lima", EMS, 17 noviembre 1928, p. 10.

22 "Decepciôn en Lima", EMS. 23 noviembre 1928, p. 15.

$2 a$ "Hoover es Esperado Hoy en el Puerto de Antotagasta donde Recibira el Homenaje de la Embrajada Boliviana que Preside el Canciller Palacios", LN, Buenos Aires, 7 diciembre 1928, p, 2 y El Presidente Electo de la Unión Recibió en el Maryland, Anclado Frente a Antofagasta, a la Embajada que le Levó el Saludo de Bolivia", LN, Buenos Aires, 9 diciembre 1928, p. 2.

24 "El Presidente Electo de la Unión Recibio en el Maryland, Anclado Frente a Antofagasta, a la Embajada que le Llevó el Saludo de Bolivia" (Hoover y Palacios Hablaron de la Confraternidad Americana), LN, Buenos Aires, 9 diciembre 1928, p, 2.

2s Chile Tributó, en Valparaiso y Santiago, un Gran Homenaje a Hoover, quien Iniciará esta Noche su Viaje a la Argentina" (El Pueblo Porteño Acogió con Aclamaciones al Viajero), LN. Buenos Aires, 11 diciembre 1928, p. 2. 
ministros de estado 26 .

En un carruaje descubierto y oficial que habia dispuesto el Gobierno de Chile, el Presidente Ibáñez acompañó a Hoover a la Embajada de Estados Unidos en el Parque Forestal, y en cuya residencia éste se hospedaria durante su permanencia en la capital chilena,

Poco rato después Hoover se presentó en el palacio de La Moneda en visita protocolar y en donde Carlos Ibáñez lo recibió en su calidad de Presidente Electo de los Estados Unidos de América y se le rindieron los honores militares de rigor y posteriormente se ofreció un almuerzo en honor del importante huésped. A su regreso a la Embajada, ya por la tarde, Hoover en compañia del Embajador Culbertson y su esposa, tomó un breve contacto con diferentes personalidades del ámbito industrial, politico y cultural del pais, quedando gratamente sorprendido por la cortesia y conocimientos industriales e internacionales de los visitantes locales. Después de un merecido descanso y un 'pequeño alto en el programa' para las primeras horas do visita en Chile, a las ocho y media de la noche del 11 de diciembre, en la Embajada estadounidense, participó de una cena de gala con la participación de importantes e ilustres invitados como del propio Ibáñez y su señora, en la cual el embajador de Estados Unidos en Chile William Culbertson, ofrecia al presidente electo de su pais un homenaje por su felicidad personal y éxito en sus futuras tareas de gobierno como también por encontrarse de paso por Chile, portando un mensaje de buena voluntad 27 .

Durante estos encuentros, los diferentes oradores chilenos plantearon a Hoover que "Si algo hemos deseado siempre es que en los Estados Unidos se conozca nuestro pais, con sus defectos y virtudes en todo su desarrollo actual, de sus tendencias y del carácter de sus habitantes. Si algo hemos lamentado es que la politica de Washington no siempre reconoció en el pasado un conocimiento cabal y un juicio seguro de esta república tan remota"28.

También y lejos de los sucesos del pasado, lo que verdaderamente importaba a los hombres de estado, comerciantes, industriales y habitantes de Chile en general era "que un presidente norteamericano visite nuestro país, se ponga en contacto con nuestros dirigentes, viva aunque sea unas horas nuestra vida y vea por sus propios ojos nuestra condición de pueblo culto, progresista y viril, nos llena de satisfacción" 29 .

En respuesta a estas elocuentes palabras Hoover agradeció en nombre de los norteamericanos y del suyo propio al gobierno y pueblo de Chile por la extraordinaria acogida y porque estaba convencido que con relaciones económicas y comerciales más prósperas ambos paises podrán continuar siendo lo que hasta la fecha han realizado en materia politica y económica: "La Historia de nuestros dos paises no es sino una cadena de esfuerzos para construir en América una nueva forma de gobierno, fundada sobre una concepción del derecho humano. Estos esfuerzos han constituido la suprema experiencia de rebelión contra los sistemas politicos sociales del Viejo Mundo. Nuestros paises han luchado juntos también por la conquista del desierto, por el desarrollo de la vida económica, por medio de los grandes descubrimientos científicos. Hemos batallado también por la elevación moral y cultural de nuestras naciones. Todavia más: en nuestras relaciones con vuestro pais no existe base alguna, afortunadamente, para una rivalidad económica o política. Nuestros intereses económicos son recíprocos. Nuestro progreso hacia la prosperidad y el bienestar general es mutuo en absoluto" 30 .

Demostrando un conocimiento bastante profundo de la situación interna del país como de su historial internacional, Hoover concluia sus palabras señalando que "He sentido especial gusto en el privilegio que vuestros hábiles ministros me han proporcionado esta mañana, al haber consentido cambiar impresiones conmigo sobre nuestra experiencia en el campo del desarrollo del progreso económico de nuestros países. La política que habéis adoptado al buscar préstamos en el extranjero, ya sea por cuenta del Gobierno o por la de una empresa privada, únicamente para trabajos reproductivos, es una restricción que bien puede adoptar el mundo entero, incluyendo las municipalidades y los estados de mi propio pais. El prestar capital para el desarrollo de obras públicas, para proporcionar medios de transporte y comunicaciones, para el desarrollo de riquezas naturales, ya sean agricolas o mineras, constituye una bendición, tanto para el prestamista como para el deudor. Las operaciones de esta naturaleza no solamente dan origen a la riqueza, que en turno se usa para

26 "Hoover Llegara Hoy a Valparatso y Santiago y Emprendera Mañana su Viaje a Buenos Aires", L.N, Buenos Aíres, 10 diciembre 1928 , p 2.

2) Chile Tributó, en Valparaiso y Santiago, un Gran Homenaje a Hoovet, quien Iniciară esta Noche su Viaje a la Argentina" (Fi Embajador Norteamericano Ofreció una Fiesta en Honor del Presidente Electo), LN, Buenos Aires, It diciembre 1928, p. 2.

28 . "Bienvenida a Hoover", EMS, 10 diciembre 1928, p. 3.

20 Ibid.

90 "El Presidente Electo de la Unión Llegará Mañana a Buenos Aires" (En el Palacio de La Moneda Ibañez Ofreció un Banquete en Honor de Hoover). LN, Buenos Aires, 12 diciembre 1928, p. 2. 
rembolsar los préstamos o las inversiones, sino que elevan a la vez las normas de vida de los empleos de las riquezas del pais prestatario"31. De lo dicho por Hoover en La Moneda de Santiago puede perfectamente encadenarse a lo que posteriormente será llamada la doctrina de la cooperación constructiva, la cual consistiria en que aquellas naciones y empresas que sepan ocupar y destinar adecuadamente sus préstamos para la producción de riquezas, podrán ser en el corto plazo también generadores de imversiones en la región y ayudar al bienestar de los pueblos.

Hoover, tras una 'suficiente' y agitada permanencia en Chile que duró un día y una noche, retomó al dia siguiente y muy temprano su itinerario latinoamericano. Al salir de Santiago el 12 de diciembre, el Presidente Electo se despedía del país diciéndole al primer mandatario Ibáñez que agradecia las bondades, cortesias y atenciones dispensadas durante su visita y que llevaria por siempre en su recuerdo el progreso, adelantos y belleza de Chile, al cual también admiraba por su hospitalidad y fineza de su pueblo ${ }^{32}$.

A los veinte y tres dias de gira latinoamericana por el Pacifico, Hoover inició su trayecto por tierra. La via maritima volvería a retomarla en el Atlántico días después.

Luego de llegar en un convoy presidencial al último punto del programa en Chile, la ciudad de Los Andes, Hoover se embarcó por la ruta del Trasandino chileno-argentino en dirección a Mendoza. Fuertemente impresionado por la belleza del paisaje cordillerano y muy contento por la posibilidad de atravesar el macizo andino latinoamericano, en Mendoza fue recibido por el Embajador de Estados Unidos en Argentina Robert Woods Bliss y una comitiva dispuesta por el Gobierno del Presidente Irigoyen.

Desde Mendoza Hoover emprendió viaje a Buenos Aires al atardecer del 12 de diciembre. Llegó a la estación de Retiro casi al anochecer del 13 de diciembre ${ }^{33}$. Al descender estaba lleno de entusiasmo y feliz por haber atravesado del Pacifico al Atlantico sin mayores problemas y por haber tenido la oportunidad de conocer en pocas horas los valles y montanias de Chile y las praderas de Argentina ${ }^{34}$.

El recibimiento de Hoover en Buenos Aires - la Nueva York del Sur - fue grandioso y su presencia voluntaria en el país estimada como histórica para "cerrar un periodo de incomprensión deplorable, para inaugurar otro de leal y positiva inteligencia"35. En Argentina Hoover era un especialista de la economia y al cual se le reconocía como "una maravilla de energía administrativa" por sus antecedentes y actuaciones en el Departamento del Comercio de Estados Unidos.

No obstante, la visita de Hoover en Argentina sirvió para que se analizaran públicamente las relaciones bilaterales. Prácticamente todos los medios de prensa informaron sobre los momentos de acercamiento y alejamiento en las relaciones de ambos paises. La idea que mayormente prevaleció en estos estudios era que Estados Unidos y Argentina han desarrollado un "progreso paralelo que deberá Ilevar a ambas naciones a una situación en que será forzoso reconocer, que habrá de ser desplegada la mayor inteligencia, justicia y mesura, para que ambos países no se consideren mutuamente como rivales y competidores, sino como amigos lo que puede redundar en un provecho común sobre la base de las relaciones comerciales"36.

Luego de tener una excelente acogida en el Congreso Nacional, recibir los homenajes del Presidente Irigoyen en el Palacio de Gobierno, entrevistarse con innumerables personeros del comercio y la política argentina, y pasear por la ciudad de Buenos Aires acompañado de su esposa y del Embajador Bliss, Hoover se embarcó en el crucero argentino 'Buenos Aires' con destino a Montevideo el 16 de diciembre ${ }^{37}$. De este modo, concluia otra visita en Sudamérica, antes de ir a Brasil.

14 "El Prestdente Electo de la Unión Llegará Mañana a Buenos Aires" (Las Grandes Riquezas de Amèrica), LN, Buenos Aires, 12 diciembre 1928, p. 2.

32 "Mr. Hoover Abandonó el Pais en la Mañana de Ayer", EMS, 12 diciembre 1928, p. 19

33. "Esta Tarde a las 18.30 boras Llegars a Retiro el Tres Especial en que Vaja el Futuro Presidente de los Estados Unidos", $L N$, Buenos Aires, 13 dielembre 1928, p. 1.

34. "Hoover Salió de Retiro en Medio de un Interminable Gentio" (Hoover Comentó con Entusiasmo el Vasto Paisaje y los Sembrados que Divisaba), LN, Buenos Aires, 14 diciembre 1928, p. 3 .

55: "Fista Tarde a las 18.30 horas Llegard a Retiro el Tren Especial en que Viaja el Futuro Presidente de los Estados Unidos"; LN, Buenos Aires, Fiditorial. 13 diciembre 1928, p. 2.

36. "En los Agasajos de Ayer, Hoover pudo Comprobar de Nuevo el Afecto Argentino", LN, Buenos Aires, 15 diciembre 1928, p, 1

37. "A Bordo del Cruceno Buenos Aires Partira esta Mañana para Montevideo el Futuro Presidente de los Estados Unidos, LN, Buenos Aires, 16 de diciembre de 1928, p. 1 y 8. También: "Hoover Partió Ayer para la Capital Uruguaya", L.N, Buenos Aires, 17 diciembre 1928, p. 1. 


\section{CONCLUSIONES}

1. La visita o gira de Hoover a Sudamérica en diciembre de 1928 fue parte de un proyecto de búsqueda de prestigio internacional antes de asumir la presidencia de Estados Unidos en marzo de 1929.

2. El Presidente Electo Hoover se sentia encabezando un nuevo proyecto interamericano sin saber exactamente hasta donde podria llevarlo.

3. La rápida visita que realizó a Centro América debemos entenderla como un primer esfuerzo para mejorar la imagen de Washington y la de un contacto de buena voluntad.

4. Las detenciones en Guayaquil y en Lima tuvieron un sentido de confraternidad más que de búsqueda de apoyos futuros en la región.

5. La entrevista concedida a una delegación boliviana en el puerto chileno de Antofagasta más bien representó un espaldarazo a las gestiones del Departamento de Estado y en particular del Secretario Frank B. Kellogg.

6. En el ámbito comercial Hoover parece estimar más libre de complicaciones tratar con Chile que con Argentina, puesto que con éste pais existirian productos equivalentes llevando a que la relación sea más competitiva y de rivalidades permanentes.

7. En el contexto Sudamericano, las visitas a Chile y Argentina habrian sido uno de los principales objetivos políticos de la gira de buena voluntad del Presidente Electo Hoover toda vez que constituirian junto a Brasil la emblemática fuerza internacional del $\mathrm{ABC}$.

\section{BIBLIOGRAFIA}

El Mercurio de Santiago, noviembre-diciembre 1928.

La Nación, Buenos Aires, Argentina, diciembre 1928.

León Wöppke, Consuelo. 2008. "¿Transición Hooveriana en la Política Hemisférica de los Estados Unidos? El Caso del Cono Sur y de los Paises Andinos", en: Estudios Norteamericanos, NN 17, Viña del Mar, pp. 119 135. 